\title{
Sublethal exposure of organophosphate pesticide chlorpyrifos alters cellular iron metabolism in hepatocytes and macrophages
}

\author{
LI SUN ${ }^{1,2}$, SHUPING ZHANG ${ }^{1}$, WENLI GUO ${ }^{1}$, WEI HE ${ }^{1}$, YI QIAN ${ }^{1}$, GUANGBO QU $^{1}$, \\ HONG JI ${ }^{2}$, HAIQIN RONG ${ }^{2}$ and SIJIN LIU ${ }^{1}$

\begin{abstract}
${ }^{1}$ State Key Laboratory of Environmental Chemistry and Ecotoxicology, Research Center for Eco-Environmental Sciences, Chinese Academy of Sciences, Haidian, Beijing 100085; ${ }^{2}$ Shandong Institute of Endocrine and Metabolic Diseases,

Shandong Academy of Medical Sciences, Jinan, Shandong 250062, P.R. China
\end{abstract}

Received February 6, 2014; Accepted August 5, 2014

DOI: $10.3892 /$ ijmm.2014.1902

\begin{abstract}
Chlorpyrifos (CPF) is commonly used for agricultural and domestic applications, and its contamination is widely detected in environmental media, and in a broad spectrum of field crops, fruits and vegetables. CPF exposure causes many adverse effects on human health including hepatoxicity, neurotoxicity and endocrine disruption. However, few studies have been conducted thus far to investigate the potential influence of $\mathrm{CPF}$ exposure on iron metabolism at concentrations that do not trigger significant cell death. Iron metabolism is concertedly governed by the hepcidin-ferroportin axis, where hepcidin is the central hormone involved in the regulation of iron absorption and release, while ferroportin is the only known iron exporter that functions by iron egress from cells. In the present study, we demonstrated that $\mathrm{CPF}$ treatment at a non-toxic concentration greatly enhanced ferroportin gene transcription in human macrophage THP-1 cells. CPF significantly inhibited hepcidin expression in human hepatocyte HepG2 cells. As a result, the intracellular labile iron pool (LIP) was largely reduced in THP-1 and HepG2 cells. The combined data of this study identified a novel finding of CPF that disrupts iron homeostasis by altering ferroportin and hepcidin expression. These findings would be useful in understanding the biological effects of $\mathrm{CPF}$ exposure, especially under relatively low and non-toxic doses.
\end{abstract}

Correspondence to: Professor Sijin Liu, State Key Laboratory of Environmental Chemistry and Ecotoxicology, Research Center for Eco-Environmental Sciences, Chinese Academy of Sciences, 18 Shuangqing Road, Haidian, Beijing 100085, P.R. China E-mail: sjliu@rcees.ac.cn

Professor Haiqin Rong, Shandong Institute of Endocrine and Metabolic Diseases, Shandong Academy of Medical Sciences, Jinan, Shandong 250062, P.R. China

E-mail: haiqinrong@126.com

Key words: chlorpyrifos, iron metabolism, ferroportin, hepcidin

\section{Introduction}

As one of the largest produced and used pesticides, chlorpyrifos $(\mathrm{CPF})$ is widely distributed in various environmental media, and in fruits and vegetables (1-3). Numerous studies have suggested that CPF may elicit adverse effects on human health (4-6). Thus far, CFP is known as an acetylcholinesterase (AChE) inhibitor, and induces significant damage to the central nervous system (7). In addition to neurological toxicities, CFP has been found to induce reactive oxygen species (ROS), and thus cause DNA damage in several organs of animal models $(8,9)$. Other studies have shown that CPF exposure is associated with developmental disorders, autoimmune disorders $(10,11)$, and even cancers, such as lung and breast cancer $(12,13)$. Moreover, histological examinations also show that CPF administration leads to substantial injury to liver, resulting in infiltration of macrophages in the portal area in an animal study (9).

Liver is the central organ governing systemic iron homeostasis, where hepcidin is primarily secreted by hepatocytes and regulates iron flux by interacting with its receptor ferroportin, which is associated with the internalization and degradation of ferroportin, thereby reducing iron egress from macrophages and iron absorption from the small intestine (14-16). Iron, an essential element, is important for a variety of biological processes $(17,18)$. Systemic iron homeostasis is deliberately modulated by the hepcidin-ferroportin regulatory axis (14,19-21). Ferroportin is the only known mammalian iron exporter, mainly expressed in epithelial cells in duodenum and macrophages, and is critical for controlling iron egress $(15,16)$. Through the hepcidin-ferroportin axis, downregulated hepatic hepcidin increases the serum iron level by promoting iron absorption from enterocytes in duodenum and iron release from macrophages (22). Increased ferroportin concentration enhances iron egress from cells, thereby decreasing intracellular iron (23).

However, studies available on CPF-conducted impairments to liver-centered iron homeostasis are currently limited, and the present literature even remains contradictory. Furthermore, little insight has been gained into potential effects of CPF on the hepcidin-ferroportin axis, and the molecular mechanisms responsible for CPF-mediated actions on iron metabolism remains to be clarified. To the best of our knowledge, in the present study, we demonstrated for the first time that CPF 
was able to disturb iron metabolism by enhancing ferroportin expression in macrophages and inhibiting hepcidin expression in hepatocytes at sublethal concentrations.

\section{Materials and methods}

Chemicals, reagents and cell culture. CPF was purchased from Shuangma Fine Chemical Co., Ltd. (Nantong, China) with the purity of $>99.99 \%$. The THP-1 human macrophage, HepG2 human hepatic carcinoma and HEK293T human embryonic kidney cell lines were obtained from the Shanghai Cell Bank of Type Culture Collection of Chinese Academy of Sciences. The cells were cultured in RPMI-1640 medium (Gibco) supplemented with $10 \%$ fetal bovine serum (FBS) and $100 \mathrm{U} / \mathrm{ml}$ penicillin/streptomycin (both from HyClone, Logan, UT, USA) in a humidified incubator (Thermo Fisher Scientific, Inc., Waltham, MA, USA) with $5 \% \mathrm{CO}_{2}$ at $37^{\circ} \mathrm{C}$. Differentiation of THP-1 cells into macrophages was induced with $1 \mu \mathrm{g} / \mathrm{ml}$ PMA (Promega, Madison, WI, USA) in complete medium for $18 \mathrm{~h}$.

Alamar blue assay. Cell viability was determined through the Alamar blue assay according to the manufacturer's instructions (Thermo Fisher Scientific, Inc.). Briefly, THP-1 and HepG2 cells were seeded in 96-well plates at a concentration of $5.0 \times 10^{3}$ cells/well. The cells were then treated with CPF for $24 \mathrm{~h}$, followed by emission detection at $590 \mathrm{~nm}$ with excitation at $530 \mathrm{~nm}$.

Western blotting. Cells were harvested and washed twice with cold phosphate-buffered saline (PBS). Total proteins were then extracted with RIPA lysis buffer (Solarbio, Beijing, China) supplemented with protease inhibitor cocktail (Roche Applied Science, San Francisco, CA, USA). Equal amounts of protein lysates (30-50 $\mu \mathrm{g} / \mathrm{sample})$ were subjected to $10 \%$ SDS-PAGE and western blot analysis was performed as described previously (24). Antibodies included anti-ferroprotin (1:500; Sigma-Aldrich, St. Louis, MO, USA) and anti-glyceraldehyde 3-phosphate dehydrogenase (GAPDH) (1:1,000; Santa Cruz Biotechnology, Inc., Santa Cruz, CA, USA). Intensity of bands was assessed with the ImageJ software (http://rsb.info.nih.gov/ij/). GAPDH was used as an internal control for normalization.

RNA extraction and RT-PCR analysis. Total RNAs were isolated from cells with TRIzol according to the manufacturer's instructions (Invitrogen Life Technologies, Carlsbad, CA, USA). The first strand of cDNA was synthesized from $2 \mu \mathrm{g}$ total RNAs using oligo(dT) primer. Primer sequences used for the PCR reaction were: hepcidin, 5'-CCTGACCAGTGGCTCTGTTT-3' (forward) and 5'-CACATCCCACACTTTGATCG-3' (reverse); GAPDH, 5'-GAAGGTGAAGGTCGGAGT-3' (forward) and 5'-GAAGATGGTGATGGGATTTC-3' (reverse). GAPDH was used as an internal control.

Luciferase reporter assays. A DNA fragment with ferroportin promoter region was subcloned into the pGL3-Promoter luciferase reporter vector (Promega) to replace the SV40 promoter. The final construct was validated by DNA sequencing (Invitrogen). Then, $0.8 \mu \mathrm{g}$ target construct plus 80 ng Renilla luciferase construct were co-transfected into HEK293T cells in 24-well plates using Lipofectamine ${ }^{\mathrm{TM}} 2000$
(Invitrogen). After $24 \mathrm{~h}$, the cells were washed with PBS, and assayed for luciferase activity using a dual-luciferase reporter assay system according to the manufacturer's instructions (Promega). Relative firefly luciferase activities were subsequently normalized to those of Renilla luciferase.

Labile iron pool (LIP) assay. Intracellular LIP was measured as previously described (25). Briefly, the cells were collected and washed following treatments, and subsequently incubated with $0.5 \mu \mathrm{M}$ calcein acetoxymethyl ester (CA-AM) (Sigma-Aldrich) for $15 \mathrm{~min}$ at $37^{\circ} \mathrm{C}$. After washing with PBS, the cells were equally divided into two groups. One group was treated with $100 \mu \mathrm{M}$ desferoxamine (DFO; Sigma-Aldrich) for $1 \mathrm{~h}$ at $37^{\circ} \mathrm{C}$, and the second group was left untreated. The cells were subjected to FACS analysis (FACSCalibur; BD Biosciences, San Jose, CA, USA), where calcein was excited at $488 \mathrm{~nm}$ and measured at $525 \mathrm{~nm}$. Intracellular LIP was obtained through deduction of the cellular fluorescence of DFO-treated cells from that of the untreated cells.

Statistical analysis. Significance of data between two groups was determined by the two-tailed independent $t$-test. One-way analysis of variance (ANOVA) was employed to assess the mean differences among groups relative to the control. Experimental data were shown as mean \pm standard error (SE). $\mathrm{P}<0.05$ was considered to indicate a statistically significant result.

\section{Results}

Cytotoxicity following CPF treatment in macrophages and hepatocytes. To examine the potential effects of CPF exposure on iron metabolism, two cardinal types of cells were employed to determine systemic iron flux, macrophages and hepatocytes $(16,26,27)$. The THP-1 human macrophage and HepG2 human hepatic carcinoma cell lines were used. In order to dissect the non-toxicity biological effects from cytotoxicity, in other words, to focus on the biological effects of CPF without exerting significant injuries on cells, concentrations that did not induce significant toxicity to cells were initially obtained. THP-1 and HepG2 cells were treated with different concentrations of $\mathrm{CPF}$ for $24 \mathrm{~h}$, and cell viabilities were measured using the Alamar blue assay. No alterations of cell viability were observed when the concentrations of CPF were $<80 \mu \mathrm{M}$ for THP-1 cells, while cell viability was reduced $>10 \%$ at $80 \mu \mathrm{M}$ $(\mathrm{P}<0.05)$ and subsequently reduced by $>30 \%$ at $100 \mu \mathrm{M}$ $(\mathrm{P}<0.05)$ (Fig. 1A). With respect to HepG2 cells, no detectable cytotoxicity occurred when the cells were treated with CPF up to $100 \mu \mathrm{M} \mathrm{CPF}$ ( $\mathrm{P}>0.05)$ (Fig. 1B). Therefore, in the present study, a non-toxic (i.e., sublethal) concentration, $20 \mu \mathrm{M}$, was selected for the subsequent experiments. Notably, the medium of $20 \mu \mathrm{M} \mathrm{CPF}$ contained $0.001 \%$ DMSO only. Medium with $0.001 \%$ DMSO caused no toxicity to cells compared to the complete blank control, and it served as a control in the present study (designated as control or cont in the text and figures).

CFP elevates ferroportin expression through transcriptional regulation. Ferroportin is the only known iron exporter in mammal animals, and its dysfunction is involved in various iron disorders, such as thalassemias and hemochromatosis $(16,27,28)$. We therefore investigated the effect of CPF treatment on 
A

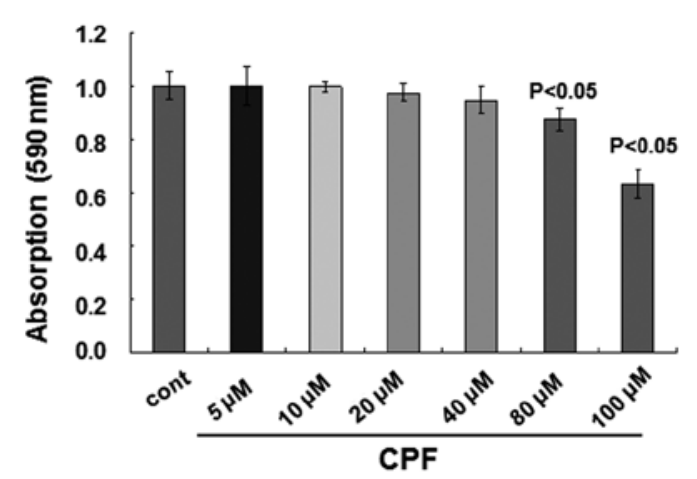

B HepG2 cells

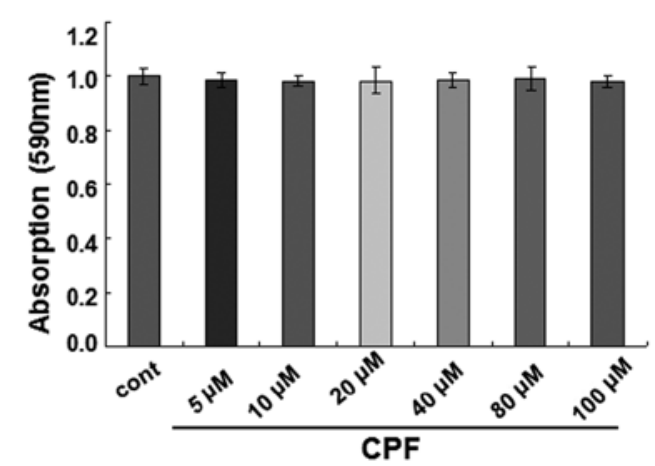

Figure 1. Cell viability assay of THP-1 and HepG2 cells treated with various concentrations of chlorpyrifos (CPF). Cell survival was assessed by Alamar blue assay in (A) THP-1 and (B) HepG2 cells up to $100 \mu \mathrm{M} \mathrm{CPF}$ for $24 \mathrm{~h}(\mathrm{n}=4-5)$.

A

Ferroportin

GAPDH

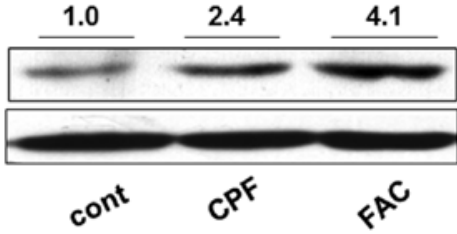

B

Ferroportin

GAPDH

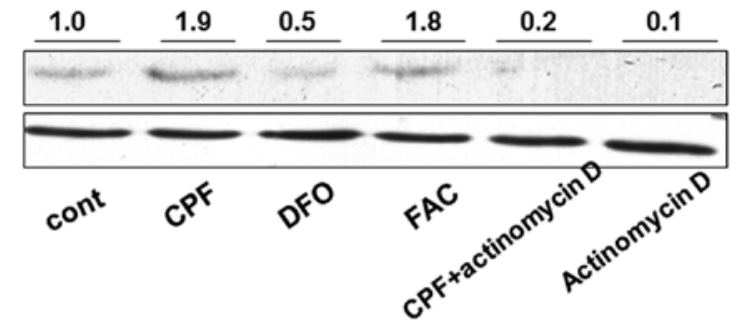

Figure 2. Concentration of ferroportin in THP-1 cells treated with chlorpyrifos (CPF) as reflected by western blot analysis. (A) Cells were exposed to $20 \mu \mathrm{M}$ CPF for $24 \mathrm{~h}$. (B) Cells were exposed to $20 \mu \mathrm{M} \mathrm{CPF}$ in the presence or absence of $1 \mu \mathrm{g} / \mathrm{ml}$ actinomycin D for $12 \mathrm{~h}$.

ferroportin concentration in THP-1 macrophages. Following treatment with $\mathrm{CPF}$ at $20 \mu \mathrm{M}$ for $24 \mathrm{~h}$, the ferroportin concentration was significantly induced by $>2$-fold in CPF-treated cells compared to the untreated cells (Fig. 2A). Ferric ammonium citrate (FAC) $(100 \mu \mathrm{M})$, as a positive control, was used to increase ferroportin concentration (29) (Fig. 2A). To investigate the mechanism underlying the increase of ferroportin concentration, a transcription inhibitor, actinomycin D, was used to attenuate global mRNA transcription. Actinomycin D treatment alone greatly reduced ferroportin concentration in THP-1 cells, suggesting marked transcriptional inhibition by actinomycin D (Fig. 2B). The increase of ferroportin concentration by $\mathrm{CPF}$ was markedly reduced when cells were simultaneously treated with actinomycin D compared to that in cells treated with CPF only (Fig. 2B). The ferroportin concentration in the cells treated with $\mathrm{CPF}+$ actinomycin $\mathrm{D}$ was greater than that in the cells treated with actinomycin D only (Fig. 2B). These results suggested that $\mathrm{CPF}$ promoted the ferroportin level through a transcriptional regulatory mechanism. FAC was used as a positive control to elevate ferroportin, and DFO $(100 \mu \mathrm{M})$, a negative control (30), was used to impede the ferroportin increase (Fig. 2B).
To substantiate the regulation of ferroportin by CPF at the transcriptional level, we performed the luciferase reporter assay to examine ferroportin expression driven by its own promoter upon CPF (Fig. 3). Following exposure to CFP at $20 \mu \mathrm{M}$ for $24 \mathrm{~h}$ in HEK293T cells, luciferase activity was measured. Compared to the control, CPF treatment increased luciferase activity by $\sim 2.5$-fold $(\mathrm{P}<0.05)$ (Fig. 3), supporting the transcriptional regulation of ferroportin by CPF. These data together identified a novel effect of CPF on promoting ferroportin expression in macrophages.

CPF inhibits hepatic hepcidin expression. To understand the biological effects of CPF on iron metabolism, hepatic hepcidin expression was investigated. As the master regulator of systemic iron homeostasis, hepcidin is predominantly secreted by hepatocytes (31). Additionally, hepcidin directs iron flow by suppressing ferroportin-conducted iron release (32). Hepcidin reduction is associated with increased iron egress and intracellular iron depletion. HepG2 cells were similarly treated with CPF at $20 \mu \mathrm{M}$. After $24 \mathrm{~h}$, we found that the hepcidin mRNA level was greatly reduced by $\mathrm{CPF}$ (Fig. 4A). The quantified data showed an $\sim 50 \%$ reduction of 
A

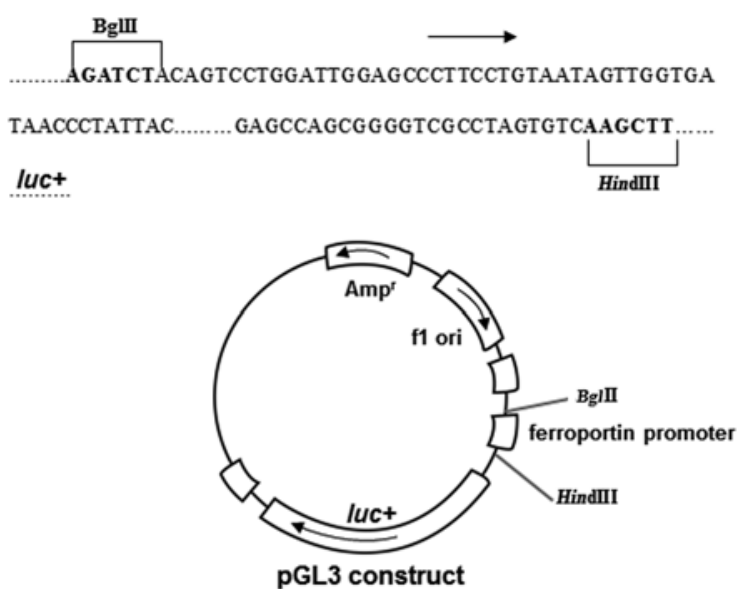

B

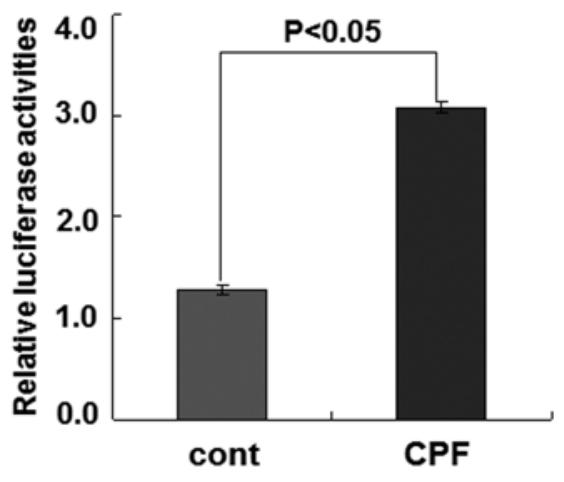

Figure 3. Chlorpyrifos (CPF) enhances the activity of ferroportin transcriptional promoter. (A) A schematic diagram of the construct bearing ferroportin promoter. By replacing SV40 promoter, ferroportin promoter was subcloned into the pGL3-promoter luciferase reporter vector using restriction endonucleases $B g l \mathrm{II}$ and HindIII. (B) Assessment of relative luciferase activities. Ferroportin promoter construct was co-transfected with Renilla luciferase plasmid into HEK293T cells, and the cells were treated with or without $20 \mu \mathrm{M} \mathrm{CPF}$ for $24 \mathrm{~h}$. Relative luciferase activities were normalized to those of the Renilla luciferase (n=4).
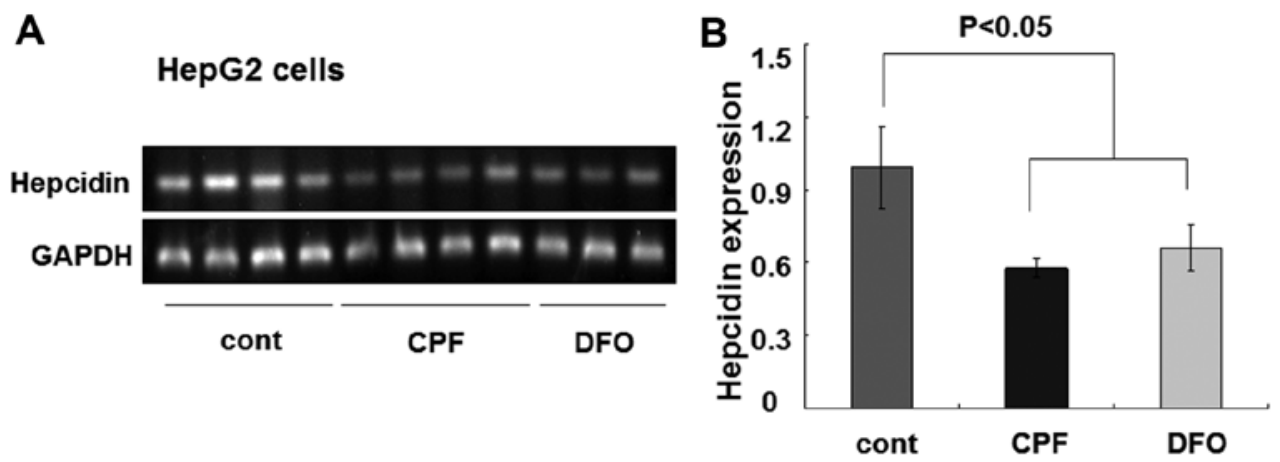

Figure 4. Hepcidin expression in HepG2 cells following exposure to chlorpyrifos (CPF). HepG2 cells were treated with (A) $20 \mu \mathrm{M} \mathrm{CPF}$ for $24 \mathrm{~h}$ followed by (B) RT-PCR analysis. The bar graph shows the quantified data for hepcidin expression $(n=4)$.

\section{A THP-1 cells}

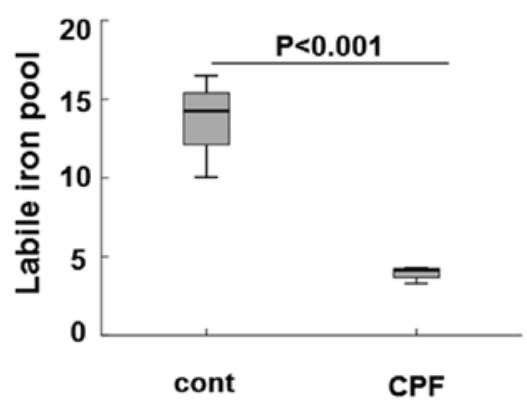

B HepG2 cells

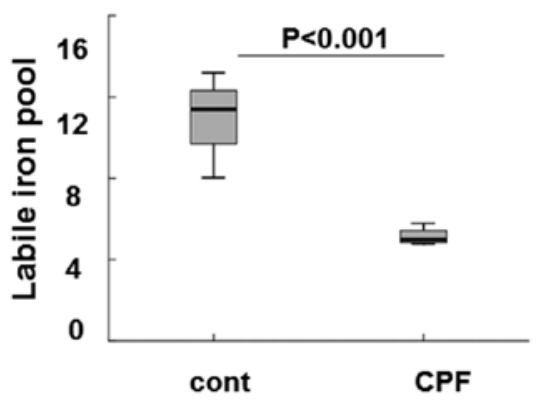

Figure 5. Labile iron pool (LIP) level in THP-1 and HepG2 cells following exposure to chlorpyrifos (CPF). (A) THP-1 and (B) HepG2 cells were treated with $20 \mu \mathrm{M}$ CPF for $24 \mathrm{~h}$ followed by the CA-AM-based LIP level assay $(\mathrm{n}=4)$.

the hepcidin mRNA level in CPF-treated cells relative to the untreated cells $(\mathrm{P}<0.05)$ (Fig. 4B). Consistent with previous studies (33), DFO was used as a control to inhibit hepcidin expression $(\mathrm{P}<0.05)$ (Fig. 4). These results demonstrated a considerable inhibitory effect of CPF on hepatic hepcidin expression.
Decreased LIP following CFP treatment in macrophages and hepatocytes. The available section of intercellular iron occurred in loosely-bound divalent iron form (i.e., LIP). It was readily and rapidly involved in the synthesis of iron-sulfur clusters, heme and other iron-containing proteins $(34,35)$. LIP was largely determined by the concentrations of iron exporter ferroportin 


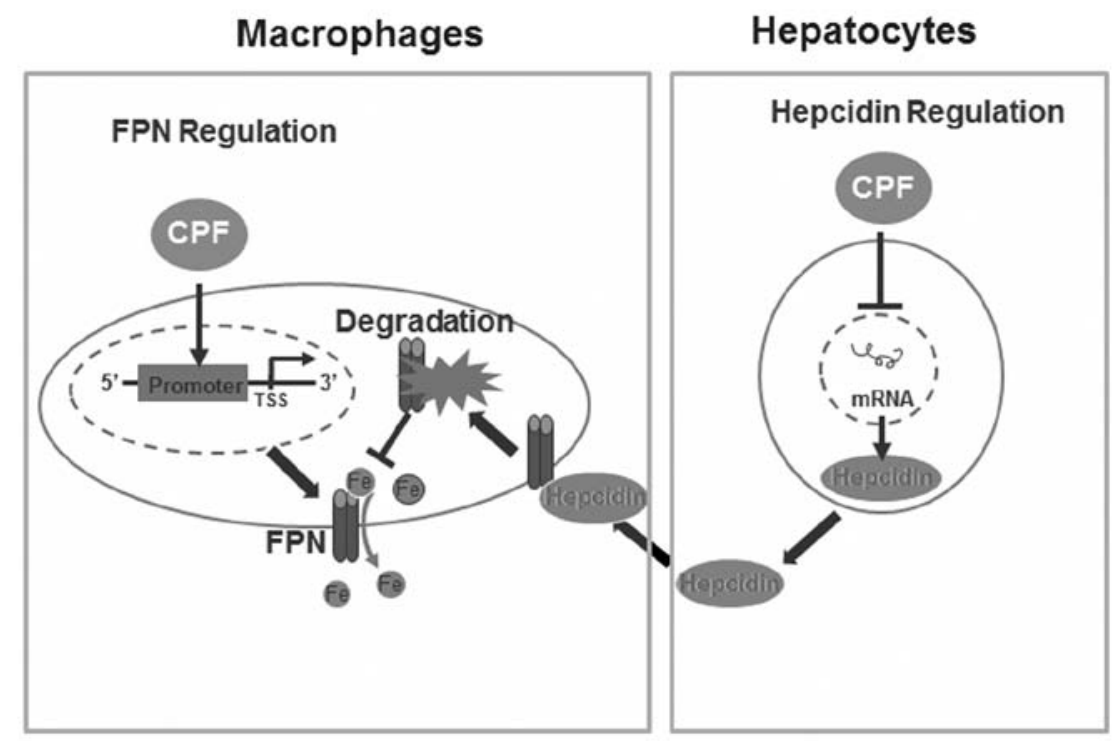

Figure 6. A schematic diagram elucidating the mechanisms by which chlorpyrifos (CPF) alters cellular iron metabolism to macrophages and hepatocytes.

and iron-storage protein ferritin (35). Thus, the intracellular iron concentration was sensitively reflected by the availability and abundance of cellular LIP. To elucidate the consequential effects on iron storage following CPF exposure in THP-1 and HepG2 cells, we assayed LIP in the two types of cells following CPF treatment at $20 \mu \mathrm{M}$ for $24 \mathrm{~h}$. As shown in Fig. 5A the level of intracellular LIP was markedly reduced by $\sim 3$-fold in THP-1 cells in CPF-treated cells relative to the untreated cells $(\mathrm{P}<0.001)$, consistent with the significant induction of ferroportin subsequent to CPF treatment (Fig. 2). The level of intracellular LIP was greatly reduced by $>2$-fold in HepG 2 cells treated with $\mathrm{CPF}$ compared to the untreated cells (Fig. 5B) $(\mathrm{P}<0.001)$, concomitant to hepcidin reduction in these cells following CPF (Fig. 4). These data signified the biological consequences of CPF on iron flow in macrophages and hepatocytes, by modulating ferroportin and hepcidin expression, respectively.

\section{Discussion}

When CPF is uptaken, it may accumulate in various organs, such as liver, kidney, ovary and uterus (36-38), with liver being one of the preferential targets (39). Long-term exposure to CPF results in significant histopathological alterations to liver, including hepatocytic vacuolation, sinusoidal dilation and focal necrosis (40). The potential non-toxic biological effects of CPF on liver, i.e., changes of biological functions without significant cytotoxicity, has been been recognized. Hepatocytes and macrophages, synergistically play a pivotal role in governing systemic iron homeostasis (41). In the present study, we identified a novel effect of CFP treatment on iron flow by altering the iron gene expression in hepatocytes and macrophages. Hepcidin expression was inhibited in HepG2 hepatocytes following CPF treatment, accompanied by reduced LIP, while ferroportin expression was elevated in THP-1 macrophages following CPF treatment, which was associated with LIP reduction.

Reticuloendothelial macrophages are the most important cells for iron storage, as they are involved in aborbing senescent red blood cells to recycle iron for erythropoiesis (42), where ferroportin, as the only known iron exporter, controls iron egress out of macrophages (43). An increased ferroportin concentration enhances iron release and reduces intracellular iron storage, as evidenced by LIP reduction $(44,45)$. In the present study, we demonstrate that CPF exposure increased ferroportin concentration in THP-1 macrophages, and this increase was mainly due to the upregulation of ferroportin transcription. As a result of the ferroportin increase, the available iron content as reflected by LIP was reduced in THP-1 macrophages. By contrast, we identified a reduced hepatic hepcidin expression in HepG2 hepatocytes following CPF treatment, which led to a reduced intracellular iron level as evidenced by LIP reduction presumably due to the hepcidin-induced degradation of ferroportin in hepatocytes. These results suggest that CPF exposure elicited significant changes to cellular iron homeostasis by regulating hepcidin and ferroportin expression in macrophages and hepatocytes. The detailed mechanisms underlying CPF-promoted activity in transcribing ferroportin and $\mathrm{CPF}-$ mediated hepcidin repression should be further investigated.

In conclusion, results of the present study have identified a novel finding of CPF in altering cellular iron homeostasis without imparing cell viability. We have demonstrated that CPF exposure significantly altered the expression of central iron genes, i.e., CPF elevates ferroportin expression in macrophages and represses hepcidin expression in hepatocytes, coupled with LIP reduction in macrophages and hepatocytes. A proposed schematic diagram deciphering the mechanisms responsible for $\mathrm{CPF}$-induced alterations to cellular iron homeostasis is presented in Fig. 6.

\section{Acknowledgements}

This study was supported by a grant under the national '973' program (grant no. 2014CB932000) and the National Natural Science Foundation of China (grant no. 21377159). We would like to thank the laboratory members for their great assistance with the experiments and reagents. 


\section{References}

1. Zhao L, Wang F and Zhao J: Identification and functional characteristics of chlorpyrifos-degrading and plant growth promoting bacterium Acinetobacter calcoaceticus. J Basic Microbiol: May 26, 2013 (Epub ahead of print).

2. Kumar Singh B, Walker A and Wright DJ: Persistence of chlorpyrifos, fenamiphos, chlorothalonil, and pendimethalin in soil and their effects on soil microbial characteristics. Bull Environ Contam Toxicol 69: 181-188, 2002.

3. Gebremariam SY, Beutel MW, Yonge DR, Flury M and Harsh JB: Adsorption and desorption of chlorpyrifos to soils and sediments Rev Environ Contam Toxicol 215: 123-175, 2012.

4. Mink PJ, Kimmel CA and Li AA: Potential effects of chlorpyrifos on fetal growth outcomes: implications for risk assessment. J Toxicol Environ Health B Crit Rev 15: 281-316, 2012.

5. Ridano ME, Racca AC, Flores-Martin J, et al: Chlorpyrifos modifies the expression of genes involved in human placental function. Reprod Toxicol 33: 331-338, 2012.

6. Singh S, Kumar V, Thakur S, et al: DNA damage and cholinesterase activity in occupational workers exposed to pesticides. Environ Toxicol Pharmacol 31: 278-285, 2011.

7. Sandahl JF, Baldwin DH, Jenkins JJ and Scholz NL: Comparative thresholds for acetylcholinesterase inhibition and behavioral impairment in coho salmon exposed to chlorpyrifos. Environ Toxicol Chem 24: 136-145, 2005.

8. Gupta SC, Mishra M, Sharma A, et al: Chlorpyrifos induces apoptosis and DNA damage in Drosophila through generation of reactive oxygen species. Ecotoxicol Environ Saf 73: 1415-1423, 2010.

9. Ojha A, Yaduvanshi SK, Pant SC, Lomash V and Srivastava N: Evaluation of DNA damage and cytotoxicity induced by three commonly used organophosphate pesticides individually and in mixture, in rat tissues. Environ Toxicol 28: 543-552, 2013.

10. Gralewicz S: Possible consequences of AChE inhibition in organophosphate poisoning. A new approach to an old problem. Med Pr 48: 469-472, 1997 (In Polish).

11. Fu Y, Li M, Liu C, et al: Effect of atrazine and chlorpyrifos exposure on cytochrome P450 contents and enzyme activities in common carp gills. Ecotoxicol Environ Saf 94: 28-36, 2013.

12. Lee WJ, Blair A, Hoppin JA, et al: Cancer incidence among pesticide applicators exposed to chlorpyrifos in the Agricultural Health Study. J Natl Cancer Inst 96: 1781-1789, 2004.

13. Ventura C, Núñez M, Miret N, et al: Differential mechanisms of action are involved in chlorpyrifos effects in estrogen-dependent or -independent breast cancer cells exposed to low or high concentrations of the pesticide. Toxicol Lett 213: 184-193, 2012.

14. Ganz T: Hepcidin and iron regulation, 10 years later. Blood 117: 4425-4433, 2011.

15. Jelić M1, Cvetković T, Djordjević V, et al: Hepcidin and iron metabolism disorders in patients with chronic kidney disease. Vojnosanit Pregl 70: 368-373, 2013.

16. Pinnix ZK, Miller LD, Wang W, et al: Ferroportin and iron regulation in breast cancer progression and prognosis. Sci Transl Med 2: 43ra56, 2010.

17. Sheftel A, Stehling O and Lill R: Iron-sulfur proteins in health and disease. Trends Endocrinol Metab 21: 302-314, 2010.

18. Frossard E, Bucher M, Machler F, Mozafar A and Hurrell R: Potential for increasing the content and bioavailability of $\mathrm{Fe}$, $\mathrm{Zn}$ and $\mathrm{Ca}$ in plants for human nutrition. J Sci Food Agric 80: 861-879, 2000.

19. Andrews NC: Forging a field: the golden age of iron biology. Blood 112: 219-230, 2008.

20. Hentze MW, Muckenthaler MU, Galy B and Camaschella C: Two to tango: regulation of Mammalian iron metabolism. Cell 142: 24-38, 2010

21. Knutson MD: Iron-sensing proteins that regulate hepcidin and enteric iron absorption. Annu Rev Nutr 30: 149-171, 2010.

22. Ganz $T$ and Nemeth E: Hepcidin and disorders of iron metabolism. Annu Rev Med 62: 347-360, 2011.

23. Donovan A, Lima CA, Pinkus JL, et al: The iron exporter ferroportin/Slc40a1 is essential for iron homeostasis. Cell Metab 1: 191-200, 2005.
24. Qu G, Zhang C, Yuan L, et al: Quantum dots impair macrophagic morphology and the ability of phagocytosis by inhibiting the Rho-associated kinase signaling. Nanoscale 4: 2239-2244, 2012.

25. Prus E and Fibach E: Flow cytometry measurement of the labile iron pool in human hematopoietic cells. Cytometry A 73: 22-27, 2008.

26. Hentze MW, Muckenthaler MU and Andrews NC: Balancing acts: molecular control of mammalian iron metabolism. Cell 117: 285-297, 2004.

27. Nemeth E, Tuttle MS, Powelson J, et al: Hepcidin regulates cellular iron efflux by binding to ferroportin and inducing its internalization. Science 306: 2090-2093, 2004

28. Singh B, Arora S, Agrawal P and Gupta SK: Hepcidin: a novel peptide hormone regulating iron metabolism. Clin Chim Acta 412: 823-830, 2011.

29. Doyard M, Fatih N, Monnier A, et al: Iron excess limits HHIPL-2 gene expression and decreases osteoblastic activity in human MG-63 cells. Osteoporos Int 23: 2435-2445, 2012.

30. Beutler E, Hoffbrand AV and Cook JD: Iron deficiency and overload. Hematol Am Soc Hematol Educ Program 2003: 40-61, 2003.

31. Park CH, Valore EV, Waring AJ and Ganz T: Hepcidin, a urinary antimicrobial peptide synthesized in the liver. J Biol Chem 276: 7806-7810, 2001.

32. Nicolas G, Viatte L, Bennoun M, Beaumont C, Kahn A and Vaulont S: Hepcidin, a new iron regulatory peptide. Blood Cells Mol Dis 29: 327-335, 2002.

33. Gehrke SG, Kulaksiz H, Herrmann T, et al: Expression of hepcidin in hereditary hemochromatosis: evidence for a regulation in response to the serum transferrin saturation and to non-transferrin-bound iron. Blood 102: 371-376, 2003.

34. Kakhlon O and Cabantchik ZI: The labile iron pool: characterization, measurement, and participation in cellular processes(1). Free Radic Biol Med 33: 1037-1046, 2002.

35. Breuer W, Shvartsman M and Cabantchik ZI: Intracellular labile iron. Int J Biochem Cell Biol 40: 350-354, 2008.

36. Nishi K and Hundal SS: Chlorpyrifos induced toxicity in reproductive organs of female Wistar rats. Food Chem Toxicol 62: 732-738, 2013.

37. Xing H, Wu H, Sun G, Zhang Z, Xu S and Li S: Alterations in activity and mRNA expression of acetylcholinesterase in the liver, kidney and gill of common carp exposed to atrazine and chlorpyrifos. Environ Toxicol Pharmacol 35: 47-54, 2013.

38. Xing H, Li S, Wang X, Gao X, Xu S and Wang X: Effects of atrazine and chlorpyrifos on the mRNA levels of HSP70 and HSC70 in the liver, brain, kidney and gill of common carp (Cyprinus carpio L.). Chemosphere 90: 910-916, 2013.

39. Acker CI, Souza AC, Dos Santos MP, Mazzanti CM and Nogueira CW: Diphenyl diselenide attenuates hepatic and hematologic toxicity induced by chlorpyrifos acute exposure in rats. Environ Sci Pollut Res Int 19: 3481-3490, 2012.

40. Tuzmen N, Candan N, Kaya E and Demiryas N: Biochemical effects of chlorpyrifos and deltamethrin on altered antioxidative defense mechanisms and lipid peroxidation in rat liver. Cell Biochem Funct 26: 119-124, 2008.

41. Evstatiev R and Gasche C: Iron sensing and signalling. Gut 61: 933-952, 2012.

42. Kong WN, Lei YH and Chang YZ: The regulation of iron metabolism in the mononuclear phagocyte system. Expert Rev Hematol 6: 411-418, 2013.

43. Zhang Z, Zhang F, Guo X, An P, Tao Y and Wang F: Ferroportin1 in hepatocytes and macrophages is required for the efficient mobilization of body iron stores in mice. Hepatology 56: 961-971, 2012.

44. Galli A, Bergamaschi G, Recalde H, et al: Ferroportin gene silencing induces iron retention and enhances ferritin synthesis in human macrophages. Br J Haematol 127: 598-603, 2004.

45. Zhang DL, Senecal T, Ghosh MC, Ollivierre-Wilson H, Tu T and Rouault TA: Hepcidin regulates ferroportin expression and intracellular iron homeostasis of erythroblasts. Blood 118: 2868-2877, 2011. 